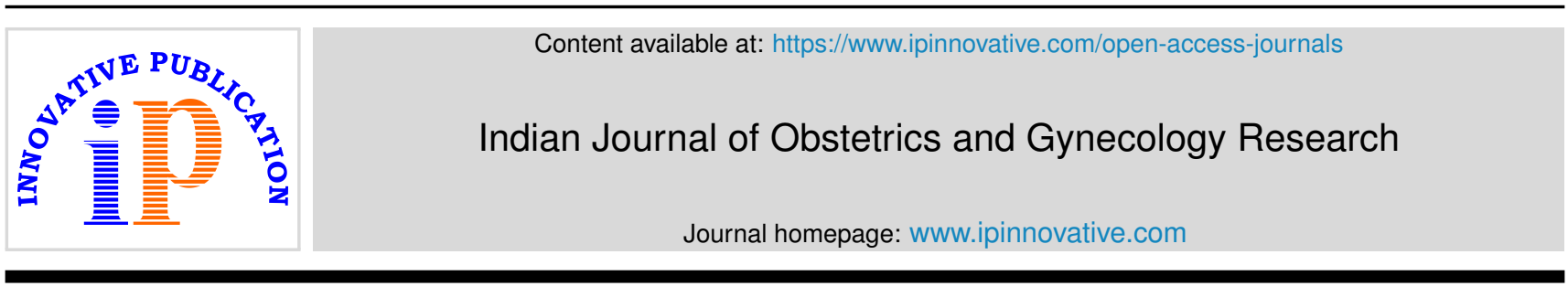

Original Research Article

\title{
Role of vaginal fluid urea and creatinine in diagnosis of premature rupture of membrane
}

\author{
Shweta Somasikta Pani*, Manasi Patnaik ${ }^{1}$, Rajlaxmi Sarangi ${ }^{1}$, Subhra Samantarey ${ }^{1}$, \\ Siddharth Behera ${ }^{1}$
}

${ }^{1}$ Dept. of Obstetrics and Gynaecology, Kalinga Institute of Medical Sciences, Bhubaneswar, Odisha, India

\section{A R T I C L E I N F O}

\section{Article history:}

Received 28-12-2019

Accepted 13-07-2020

Available online 12-09-2020

\section{Keywords:}

Premature rupture of membrane

Vaginal fluid

Urea

Creatinine

\begin{abstract}
A B S T R A C T
Introduction: Pre labour rupture of membrane is associated with an increased maternal and perinatal morbidity and mortality. So confirmatory diagnosis is essential before initiating the definitive treatment of PROM.

Materials and Methods: This study was a cross sectional, case control, hospital based study. All pregnant women having gestational age 28 to 42 weeks are divided into 3 groups as per criteria. Each group has 30 pregnant women group 1 and group 2 are the cases studied and group 3 is control group. Data analysis was done by ANOVA test, kurskal Wallis Test. Difference between two groups are analysed using Man whitney's U test.

Results: The level of vaginal fluid urea and creatinine came out to be higher in group 1 (confirmed cases) than group 2 (suspected cases) and group 3 (controls).

Conclusion: Detection of urea and creatinine in vaginal fluid is easy, safe and cost effective method to diagnose PROM.

(C) 2020 Published by Innovative Publication. This is an open access article under the CC BY-NC license (https://creativecommons.org/licenses/by-nc/4.0/)
\end{abstract}

\section{Introduction}

Preterm birth is one of the important maternal health issue. Rates are raising in developed and developing countries and it remains the most important determinant of adverse fetal outcome. When the membrane ruptures spontaneously before the onset of labour, it is called pre labour rupture of membrane. ${ }^{1}$ And when the membrane ruptures before 37 completed weeks it is called pre term PROM i.e PPROM. ${ }^{1}$ The incidence of term PROM is about $10 \%$ and that of preterm pre labour rupture of membrane is $3 \%$ globally. ${ }^{1}$ Usually labour sets in within 12 to 24 hours after rupture of membrane. ${ }^{1}$ Spontaneous onset of labour in term PROM is $86 \%$ and in PPROM is around $40 \%$. There is no definitive method to diagnose a PROM. Method for diagnosis is mainly from maternal history of watery discharge and on speculum examination seeing pool of amniotic fluid in posterior fornix. The test taken in this studyto confirm

\footnotetext{
* Corresponding author.

E-mail address: somasikta@gmail.com (S. S. Pani).
}

leaking is based on the principal that amniotic fluid contain urea and creatinine of fetal urine. Normal vagina does not contain urea and creatinine so only when the membranes rutures urea and creatinine is detected in vagina.

\section{Materials and Methods}

This study was undertaken in the Department of Obstetrics and Gynaecology of Kalinga institute of medical sciences after taking approval of the institutional Ethics Committee. An informed consent in the patients own language was taken from all pregnant women who participated in the study. The sample size was calculated based on accuracy level of diagnosis of PROM by the measurement of vaginal fluid urea and creatinine and on feasibile. Pregnant women between the ages 19 to 35 years with singleton live fetus (without any abnormality) coming to the labour room and willing to participate in the study were taken into our study. Gestational age of pregnancy of the women in our study was in between 28 and 42 completed weeks. 
Women with multiple gestation, fever, tachycardia and pain abdomen or lower uterine tenderness were excluded from the study. A detailed history of the nature and type of discharge per vagina of the pregnant women participating in the study was taken. All the participants underwent general examination, abdominal examination and sterile per speculum examination to look for any fluid coming out of the cervix or pooling of fluid in the posterior fornix. If amniotic fluid was meconium stained or blood stained then these patients were excluded from this study. So with the above criteria 90 pregnant women were taken in our study. These women were divided into three groups.

Group 1 - Women with complains of leaking per vagina, confirmed by visualization of clear liquid either in posterior fornix or coming out from the cervix by per speculum examination.

Group 2 - Women with history of leaking per vagina but no demonstrable active leaking on speculum examination, even after valsalva maneuvers (women with suspected PROM).

Group 3 - All the pregnant women in our study who gave no history of leaking per vagina (women with no PROM). This was taken as the Control group.

\subsection{Procedure}

While doing the speculum examination $5 \mathrm{ml}$ of sterile saline was pushed into the vagina with a disposable $5 \mathrm{ml}$ syringe and $3 \mathrm{ml}$ of the same fluid was aspirated back in the same sitting and sent for biochemical study in a sterile container. In Group 1 where active leaking was present, the pooled amniotic fluid was first cleaned with a sterile gauze before pushing the normal saline. The collected sample was sent to the Central Laboratory of KIMS for analysis. This sample was first centrifuged at $3000 \mathrm{rpm}$ for 10 minutes and then processed. Urea was analysd by the Urease method and Creatinine by the Jaffe's alkaline Picrate method. Tests were performed in the Auto-analyser and values were noted for analysis.

\subsection{Statistical analysis}

Kolmogorov-Smirnov test of normality was applied to all measured variables. Data were described as mean and standard deviation (for numeric parametric variables); median and interquartile range (for numeric non-parametric variables). Difference between the three groups was analyzed using one-way ANOVA test (for numeric parametric variables) and KurskalWallis test (for numeric non-parametric variables). Difference between two groups was analyzed using Mann-Whitney's U-test (for numeric non-parametric variables). Receiver operator characteristics (ROC) curves were constructed to identify cut off of vaginal fluid urea and creatinine to predict PROM. All test were considered significant at 5\%level of significance. Statistical analysis of data was performed using stata15.1,stata corp (texas, USA).

\section{Result}

All the 90 women taken in our study were divided into 3 groups according to age. Group 1, 2, 3 had 18,4 and 15 patients respectively. Of the 90 women in our study majority of them were primigravida, In all the 3 study group but as shown in Table 2, there was no significant difference in distribution with regards to gravid.

Patients were divided into 3 groups with regards to gestational age at the time of first examination in labour room, maximum number of patients were within the gestational age 34 to 37 weeks in group 1 and group 2 patients. (Table 3) Maximum no of patients in the control group were at term pregnancy (38 to 42 weeks). There were a total of $62(68.89 \%)$ primigravid and 28 (33\%) multigravida. Thus there was significant difference in gestational age ( $\mathrm{p}$ value) across the three study groups. Oligohydramnious was seen in all the confirmed (group 1) and suspected PROM cases (group 2) the mean value of AFI in confirmed cases of PROM is $4.6+\_1.2 \mathrm{~cm}$, mean value of AFI in suspected cases is $4.3+\_1.6 \mathrm{~cm}$ and mean value in controls is $10+\_1.7 \mathrm{~cm}$ (Table 4). Vaginal fluid urea and creatinine value was higher in women with confirmed PROM than women with suspected PROM and those of control group. In our study the cut off point of vaginal fluid urea and creatinine for definitive diagnosis of PROM is $8 \mathrm{mg} / \mathrm{dl}$ and $0.2 \mathrm{mg} / \mathrm{dl}$ respectively. Urea value has sensitivity of $93.3 \%$ and specificity of $100 \%$ and value of creatinine has sensitivty and specificity $100 \%$ for both. These were the results of group 1 (confirmed cases).

In group 2 (suspected cases) the cut off value of urea and creatinine is $3 \mathrm{mg} / \mathrm{dl}$ and $0.2 \mathrm{mg} / \mathrm{dl}$ respectively for diagnosis of PROM. Urea value has sensitivity of $70 \%$ and specificity of $93.3 \%$. For creatinine value both sensitivity and specificity both are $100 \%$. (Table 5)

Accuracy of vaginal fluid urea and creatinine concentrations for diagnosis of suspected PROM. (Between group 2 and group 3 )

\section{Discussion}

A case presenting with PPROM/PROM to the labour room always puts the obstetrician in a dilemma. A balanced decision is needed keeping in mind the risks of prematurity on termination and the adverse outcome of the fetus and mother in continuing the pregnancy. The tests available for diagnosing rupture of membranes are vaginal fluid nitrazine test, vaginal fluid alpha feto- protein, vaginal fluid insulin like growth factor binding protein 1, fetal fibronectin test, vaginal fluid human chorionic gonadotropin, vaginal fluid prolactin, vaginal fluid placental alpha microglobulin. But none of the said tests are easily available in all centres or are 
Table 1: Age

\begin{tabular}{lccc}
\hline Age & Group -1 & Group -2 & Group -3 \\
$19-25$ & 18 & 14 & 15 \\
$26-30$ & 5 & 9 & 9 \\
$30-35$ & 7 & 7 & 6 \\
\hline
\end{tabular}

Table 2: Parity

\begin{tabular}{lccc}
\hline Parity & Group $-\mathbf{1}$ & Group -2 & Group -3 \\
Primi & 21 & 25 & 16 \\
Multi & 9 & 5 & 14 \\
\hline
\end{tabular}

Table 3: Gestational age

\begin{tabular}{lccc}
\hline Gestational age & Group -1 & Group -2 & Group -3 \\
$28-33$ & 8 & 3 & 3 \\
$34-37$ & 21 & 18 & 12 \\
$38-42$ & 1 & 9 & 15 \\
\hline
\end{tabular}

Table 4: Difference between 3 groups amniotic fluid index (AFI)

\begin{tabular}{lcccc}
\hline $\begin{array}{l}\text { Vaginal fluid } \\
\text { concentration }\end{array}$ & $\begin{array}{c}\text { Group I } \\
\text { Confirmed prom }(\mathbf{n}=\mathbf{3 0})\end{array}$ & $\begin{array}{c}\text { Group II } \\
\text { Suspected prom }(\mathbf{n}=\mathbf{3 0})\end{array}$ & $\begin{array}{c}\text { Group III } \\
\text { No prom }(\mathbf{n}=\mathbf{3 0})\end{array}$ \\
AFI & $4.67 \pm 1.21$ & $4.37 \pm 1.16$ & $10.03 \pm 1.73$ & P Value \\
\hline
\end{tabular}

Table 5: Accuracy of vaginal fluid urea and creatinine concentrations for diagnosis of confirmed PROM. (Between group 1 and group 3)

\begin{tabular}{lcccc}
\hline Vaginal fluid concentration & Sensitivity & Specificity & PPV & NPV \\
Urea $\geq 8 \mathrm{mg} / \mathrm{dl}$ & $93.1 \%$ & $100 \%$ & $90.0 \%$ & $96.7 \%$ \\
Cretinine $\geq 0.2 \mathrm{mg} / \mathrm{dl}$ & $100 \%$ & $100 \%$ & $98.3 \%$ & $98.3 \%$
\end{tabular}

Table 6: Accuracy of vaginal fluid urea and creatinine concentrations for diagnosis of suspected PROM. (Between group 2 and group 3)

$\begin{array}{lcc}\text { Vaginal fluid concentration } & \text { Sensitivity } & \text { Specificity } \\ \text { Urea } \geq 3 \mathrm{mg} / \mathrm{dl} & 70 \% & 100 \% \\ \text { Cretinine } \geq 0.2 \mathrm{mg} / \mathrm{dl} & 93.3 \% & 100 \%\end{array}$

routinely performed. Nitrazine test though easy to perform has high false negative value. ${ }^{2}$

In our study measurement of vaginal fluid urea and creatinine in confirmed cases had a sensitivity of $93.3 \%$ and specificity of $100 \%$ and in suspected cases (Group 2) showed a sensitivity of $70 \%$ and specificity of $100 \%$.

As ultrasonography was available in the labour room AFI could also be recorded at the time of admission. The finding of oligohydramnios in Group 1 and Group 2 corroborated well with vaginal fluid urea and creatinine level for confirming the diagnosis of PROM. We were able to confirm the diagnosis of PROM in Group 2 also when both these findings were taken together.

Gada et al in the year 2018 studied the reliability of vaginal fluid urea and creatinine levels for the diagnosis of PROM. This study showed that there was a significant difference in the levels of creatinine and urea in the studied groups. The creatinine level was $0.64,0.28$ and 0.14 in confirmed, suspected and controls respectively. ${ }^{3}$ The urea levels were 29.67, 5.51 and $1.68 \mathrm{mg} / \mathrm{dl}$ in confirmed, suspected and controls respectively. ${ }^{3}$ These findings are similar to that obtained in our study where the highest levels of urea and creatinine were seen in the confirmed or Group - 1 cases. Levels of both urea and creatinine were almost insignificant in the control group.

Begum et al in year 2017 did a prospective study on vaginal fluid urea and creatinine in diagnosis of premature rupture of membrane in resource limited community settings. ${ }^{4}$ In the study 100 pregnant females were recruited of which 50 were cases of PROM and 50 were controls. In statistical analysis significance difference was seen between value of urea and creatinine in group 1 (cases) and group 2 (control). The mean value of urea was $12.6+\_4.8$ and mean value of creatinine was $0.67+\ldots .3$ in Group $1 .{ }^{4}$ The mean value of creatinine was $0.16+-.09$ and urea was $2.12+\_1.31$ in control group. They found that the mean value of urea and creatinine in study group was higher than the control group. ${ }^{4}$ Similarly our study also showed significantly higher value of urea and creatinine in case PROM compared to the control group. In our study urea level was $8 \mathrm{mg} / \mathrm{dl}$ and 
creatinine level was in confirmed $0.2 \mathrm{mg} / \mathrm{dl}$ group.

Sabee et al studied 96 women and divided them into 3 groups as in our study. They found vaginal fluid urea and creatinine level were significantly higher in women of confirmed group compared to suspected and control group. ${ }^{5}$ These finding are exactly similar to the results obtained in your study.

Saeed et al in 2011 did a study on predictive value of vaginal fluid urea and creatinine in cases of PPROM. Confirmed PROM group has higher level of vaginal fluid urea and cretinine when compared to unconfirmed group with very high statistically significant difference $(\mathrm{p}<$ $0.001)^{6}$ our study too showed significantly higher vaginal fluid urea and creatinine in confirmed cases group ( $p$ $<0.001$ )

Kariman et al in year 2013 also studied vaginal fluid urea and creatinine in confirmed and unconfirmed cases of PROM. They concluded that estimation of creatinine level had a higher diagnostic value for PROM. ${ }^{7}$ Creatinine estimation in our study both in suspected and confirmed group has $100 \%$ sensitivity and specificity.

Zanjani et al studied the reliability of vaginal fluid creatinine for diagnosis of PROM in year 2012. They concluded that vaginal fluid creatinine is a simple, reliable, rapid and inexpensive method for diagnosing PROM. ${ }^{8}$ Creatinine estimation in our study also prove to be superior to urea in PROM cases to confirm leaking.

\section{Conclusion}

Pre- labour rupture of membrane remains one of the diagnostic problems in obstetric practice. The aetiology of PROM is multifactorial and in some cases yet unclear. Managment of PROM depends on gestational age and onset of complications. Diagnosis of PROM is yet another challenge to obstetrician as traditional method of diagnosis has higher rates of false negative results.

Measurement of vaginal fluid urea and creatinine concentration is easy, cost effective and can be performed without much technical assistance.

\section{Source of Funding}

None.

\section{Conflict of Interest}

None.

\section{References}

1. Holland and Brews 4th Edition Chapter No 55. .

2. Tigga M, Malik S. Comparative analysis of four biomarkers in diagnosing premature rupture of membranes and their correlation with onset of labour. Int J Reprod Contracept Obstet Gynecol. 2015;4:10705 .

3. Gada MS, Gaied AMA, Eikhouly NI. Galal Khalaf AM. Urea and creatinine in diagnosis of premature rupture of membrane. Menoufia Med J. 2018;31:52-6.

4. Begum J, Samal SK, Ghose S, Niranjan G. Vaginal Fluid Urea and Creatinine in the Diagnosis of Premature Rupture of Membranes in Resource Limited Community Settings. J Fam Repr Heal. 2017;11:439.

5. El-Sabee H, Swidan K, Ezzat A. Vaginal Fluid Urea and Creatinine as Biomarkers of Prelabor Rupture of Membranes (PROM). Med J Cairo Univ. 2015;83:173-9.

6. Kariman N, Afrakhte M, Hedayati M, Fallahian M, Majd H. Diagnosis of Premature Rupture of Membranes by Assessment of Urea and Creatinine in Vaginal Washing Fluid. Iran J Reprod Med. 2013;11:93100.

7. Zanjani MS, Haghighi L. Vaginal fluid creatinine for the detection of premature rupture of membranes. J Obstet Gynaecol Res. 2012;38(3):505-8.

8. Mohamed A, Mostafa W. The value of measurement of vaginal urea, ceatinineand beta HCG in the diagnosis of premature rupture of membranes. KAJOG. 2011;2:41-7.

\section{Author biography}

Shweta Somasikta Pani MS Obs \& Gyn

Manasi Patnaik MS Obs \& Gyn

Rajlaxmi Sarangi MD Biochemistry

Subhra Samantarey MD Obs \& Gyn

Siddharth Behera DNB Radiodiagnosis

Cite this article: Pani SS, Patnaik M, Sarangi R, Samantarey S, Behera $\mathrm{S}$. Role of vaginal fluid urea and creatinine in diagnosis of premature rupture of membrane. Indian J Obstet Gynecol Res 2020;7(3):398-401. 